\title{
Numerical research of the perforation of targets by ice impactors at different temperatures
}

\author{
Gennady N. Bogomolov ${ }^{1, *}$, Victor P. Glazyrin ${ }^{1}$, and Maxim Yu. Orlov ${ }^{1}$ \\ ${ }^{1}$ National Research Tomsk State University, 634050 Tomsk, Russia
}

\begin{abstract}
In the present work, the process of interaction of ice impactors of spherical shape with targets of aluminum alloy and asbotextolite has been numerically investigated. The initial temperature of the impactors was set from 0 to $50^{\circ} \mathrm{C}$. The speed of the impactors was set in the range from $500 \mathrm{~m} / \mathrm{s}$ to $1500 \mathrm{~m} / \mathrm{s}$. The behavior of the medium was described from the general positions of the mechanics of continuous media and corresponded to modern concepts of the destruction of solids under load. The material was considered as isotropic, elastoplastic, compressible, porous medium. The equation of state is chosen in the Walsh form. The problem was solved in a two-dimensional formulation for the case of axial symmetry. The calculations were carried out using the Lagrangian technique of computer simulation, which takes into account the fragmentation of the material in shear and shear failure. The current configurations of the impactor-target and the velocity of the leading fragments are obtained when penetrating the targets.
\end{abstract}

\section{Introduction}

The relevance of research into the interaction of ice impactors of spherical shape with targets of aluminum alloy and asbestos cloth is caused by the extensive use of the targets in question when creating new models of aviation and rocket and space technology. In $[1,2]$ numerical results were obtained on the process of pulsed loading of ice at a certain fixed temperature. However, in terrestrial conditions $[3,4]$ the ice can be kept at a temperature of $0^{\circ} \mathrm{C}$ and up to $-60^{\circ} \mathrm{C}$. Consequently, the mathematical model of ice behavior should take into account the dependence of strength physical-mechanical characteristics on temperature.

In the present paper, the problem of the interaction of ice impactors of a spherical shape with targets of an aluminum alloy and asbotextolite is solved. Impactors are at different temperatures. The behavior of materials is described by the basic system of equations of continuum mechanics, i.e. Equations of continuity, motion and energy, written in independent Lagrangian variables [5, 6]. The problem was solved in a two-dimensional formulation for the case of axial symmetry. The current configurations of the impactortarget and the velocity of the leading fragments are obtained when penetrating the targets.

*Corresponding author: glvp@list.ru 
This approach makes it possible to predict the behavior of promising impact-resistant protections in order to facilitate the search for the optimal design variant.

\section{Mathematical model}

The physical formulation of the problem is formulated as the shock interaction of spherical ice bodies with three types of targets equivalent in mass. The initial velocity of the impactor is directed along the axis of symmetry normal to the obstacle plane and is set equal to $500 \mathrm{~m} / \mathrm{s}, 1000 \mathrm{~m} / \mathrm{s}$ and $1500 \mathrm{~m} / \mathrm{s}$. The initial temperature of the impactors was set equal to $-15^{\circ} \mathrm{C}$ and $-50^{\circ} \mathrm{C}$. The radius of the impactor is $0.5 \mathrm{~cm}$, the density is $0.92 \mathrm{~g} / \mathrm{cm}^{3}$, the bulk modulus is $8.4 \mathrm{GPa}$. The first type of target is made of aluminum alloy AMG-6, $0.5 \mathrm{~cm}$ thick. The second type of target is Asbotextolite (AsT), $0.875 \mathrm{~cm}$ thick. The target of the third type is two-layer. The first layer is AsT, $0.438 \mathrm{~cm}$ thick. The second layer is an AMG-6 alloy with a thickness of $0.25 \mathrm{~cm}$. The density of the AMG-6 alloy is $2.85 \mathrm{~g} / \mathrm{cm}^{3}$, the bulk modulus is $76.5 \mathrm{GPa}$. The density of AsT is $1.6 \mathrm{~g} / \mathrm{cm}^{3}$, the bulk modulus is 4.5 $\mathrm{GPa}$. The remaining physicomechanical characteristics of the materials in question, which are necessary for the calculation, are taken from $[3,4,6]$.

We shall assume that the strength characteristics of ice depend on temperature in the following manner [3, 4]:

$$
f_{i}=f_{\text {io }}\left(1+\frac{1}{f_{\text {io }}} \frac{\partial f}{\partial t} \Delta T\right)=f_{\text {io }}+a_{i} \Delta T,
$$

where $f_{i}$ - shear modulus $\mu_{0}$, yield stress $\sigma_{\mathrm{To}}$, tension spall $\sigma_{\mathrm{ko}}$ and critical work of plastic deformation $\mathrm{A}_{\mathrm{o}} ; f_{i o}$ - initial values of these characteristics. Constants $a_{i}$ determined from experimental data $[3,4,7]$ and the condition of the vanishing ice at zero temperature. The following values of strength characteristics used in the calculations:

$\mu_{0}=3,1 \mathrm{MPa} ; \sigma_{\text {то }}=1.85 \mathrm{MPa} ; \sigma_{\mathrm{ko}}=3.06 \mathrm{MPa} ; \mathrm{A}_{\mathrm{o}}=0.3 \mathrm{KJ} / \mathrm{kg} ; \mathrm{a}_{1}=0.035 \mathrm{MPa} /$ degree; $\mathrm{a}_{2}=$ $0.030 \mathrm{MPa} /$ degree; $\mathrm{a}_{3}=0.030 \mathrm{MPa} /$ degree; $\mathrm{a}_{4}=0.02 \mathrm{KJ} /(\mathrm{kg} \times \mathrm{deg})$.

The medium under consideration is assumed to be compressible, isotropic, mass forces, internal heat sources and thermal conductivity are absent. Shock-wave phenomena, as well as the formation of detachment and shear destruction, are taken into account. The stress tensor is divided into deviator and spherical components. To determine the hydrostatic pressure, the equation of state in the Walsh form is used. The deviator components of the stress tensor are based on the model of elastoplastic flow based on the Prandtl-Reis equations associated with the Mises yield condition [5, 8, 9].

\section{Numerical simulation}

Numerical analysis of the processes of deformation and destruction of materials of impactors and obstacles was carried out using a software package simulating the nonstationary motion of a compressible elastoplastic, porous medium, taking into account the detachment and shear nature of the fractures, with the possibility of the appearance of new free surfaces, including dividing the body under consideration into separate fragments $[1,5]$.

The initial conditions in problems of the shock interaction of solids for the unperturbed state assume that the stress components, the pressure, and the internal energy are zero. The boundary conditions on the contact surfaces allow their sliding without friction. The solution of the given boundary-value problem is carried out in a two-dimensional formulation for axial symmetry by the Johnson Lagrangian method, generalized to the case 
of large deformations and fracture of the material into fragments by introducing the mechanisms of splitting the calculated nodes and destroying the calculated elements into the computational part of the model $[5,9]$.

\section{Results}

The calculated current configurations of the target striker in the interaction of the ice ball with the obstacle from AMG-6 with an initial velocity of $500 \mathrm{~m} / \mathrm{s}$ are shown in figure 1 . It is obtained that already for $4 \mu$ s the impactor is covered by a dense network of cracks, and by $100 \mu \mathrm{s}$ it is completely destroyed into small Fragments. In this case, the target is practically not deformed. Figure $1 \mathrm{~b}$ shows the results of calculating an analogous variant with an initial shock velocity of $1000 \mathrm{~m} / \mathrm{s}$. It can be seen that also for 4 microseconds the impactor is covered with a dense network of cracks, and for 100 microseconds it is completely destroyed into small fragments. The barrier is considerably deformed and destroyed both from the front and from the rear. However, there is no through penetration either at a temperature of $-15^{0} \mathrm{C}$ or $-50^{\circ} \mathrm{C}$.

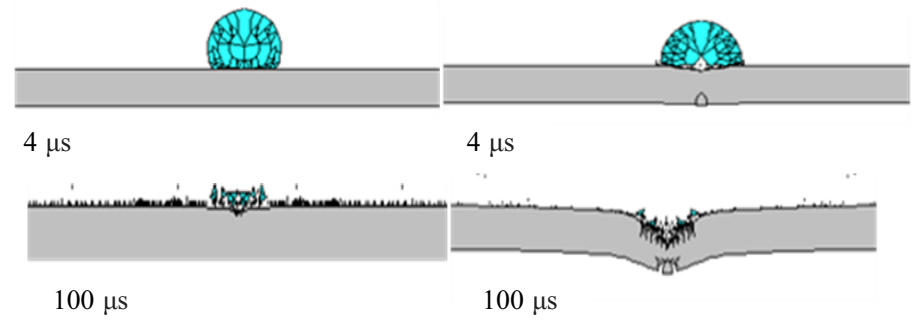

(a)

(b)

Fig. 1. Results of calculation of the interaction with targets projectiles at $T=-15^{0} \mathrm{C} ; \mathrm{a}-\mathrm{V}_{\mathrm{o}}=500 \mathrm{~m} / \mathrm{s}$, $\mathrm{b}-\mathrm{Vo}_{\mathrm{o}}=1000 \mathrm{~m} / \mathrm{s}$.

The calculated current configurations of the impactor -target for obstacles from AMG-6 with an initial velocity of $1500 \mathrm{~m} / \mathrm{s}$ are shown in figure $2 \mathrm{a}$. On the 4 th microsecond, the impactor is practically destroyed. There are also disruptions in the target. At the time of 10 microseconds, the collapsed impactor penetrated the target by half its thickness. At a time of 30 microseconds, a through hole exists in the target, and the leading fragments move with a constant velocity equal to $510 \mathrm{~m} / \mathrm{s}$ for the variant with the impactor temperature $\mathrm{T}=$ $-15^{\circ} \mathrm{C}$. For the variant with the impactor temperature $\mathrm{T}=-50^{\circ} \mathrm{C}$, the leading fragments move at a velocity equal to $565 \mathrm{~m} / \mathrm{s}$.

Figure $2 \mathrm{~b}$ shows the results of calculating the variant with an obstacle from AsT and an initial strike velocity of $500 \mathrm{~m} / \mathrm{s}$. It is seen that for $6 \mu$ s the impactor is rapidly destroyed, and by $30 \mu \mathrm{s}$ it is completely destroyed into small fragments. The barrier has fractures of a cross-cutting nature. At $100 \mu \mathrm{s}$, a small fragment of the rear surface of the obstacle is observed moving at a speed of $100 \mathrm{~m} / \mathrm{s}$. For a variant with a impactor temperature $\mathrm{T}=-$ $50^{\circ} \mathrm{C}$, the velocity of such a fragment is $112 \mathrm{~m} / \mathrm{s}$. 


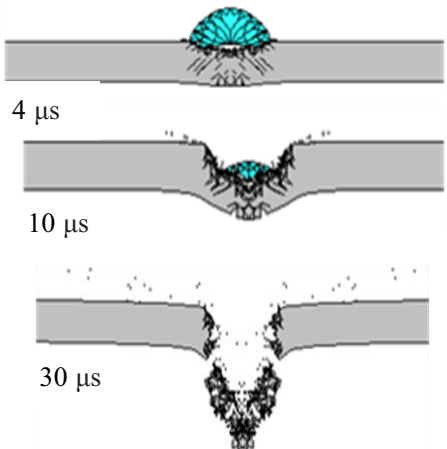

(a)
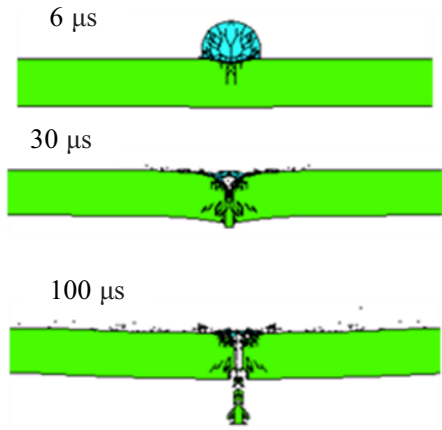

(b)

Fig. 2. Results of calculating the process of interaction of impactors with targets at $\mathrm{T}=-15^{\circ} \mathrm{C}$; a targets AMG-6, Vo $=1500 \mathrm{~m} / \mathrm{s}, \mathrm{b}-$ AsT targets, $\mathrm{Vo}=500 \mathrm{~m} / \mathrm{s}$.

Figure $3 \mathrm{a}$ shows the results of calculating an analogous variant with an initial impactor velocity of $1000 \mathrm{~m} / \mathrm{s}$. It can be seen that in the process of interaction the impactor completely collapsed. At $100 \mu \mathrm{s}$, the barrier has a through hole, and the leading fragments move at a constant velocity of $345 \mathrm{~m} / \mathrm{s}$. At the impactor temperature $\mathrm{T}=-50^{\circ} \mathrm{C}$, the leading fragments move at a velocity equal to 378 m/s. For the variant with an initial velocity of $1500 \mathrm{~m} / \mathrm{s}$ (figure $3 \mathrm{~b}$ ), it turned out that the hole diameter is somewhat larger than in the previous case, and the leading fragments move with a constant velocity equal to $600 \mathrm{~m} / \mathrm{s}$. At the impactor temperature $\mathrm{T}=-50^{\circ} \mathrm{C}$, the leading fragments move at a velocity equal to $655 \mathrm{~m} / \mathrm{s}$. The results of the calculation of the process of interaction of impactors with two-layered targets from AsT $(0.438 \mathrm{~cm})$ and AMG-6 $(0.25 \mathrm{~cm})$ are shown in Fig. 4. It can be seen that at an interaction velocity of $500 \mathrm{~m} / \mathrm{s}$ (figure 4a), the asbotextolite layer is destroyed in the center, the layer from AMG-6 has small deformations.

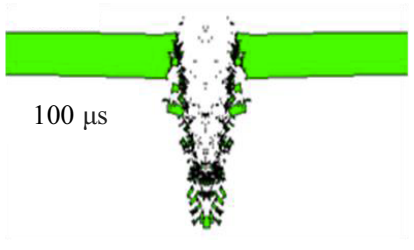

(a)

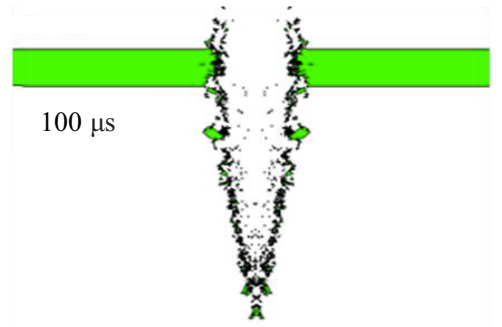

(b)

Fig. 3. Results of calculating the process of interaction of impactors with targets at $\mathrm{T}=-15^{\circ} \mathrm{C}$ with targets from AcT; $\mathrm{a}-\mathrm{V}_{\mathrm{o}}=1000 \mathrm{~m} / \mathrm{s}, \mathrm{b}-\mathrm{V}_{\mathrm{o}}=1500 \mathrm{~m} / \mathrm{s}$.

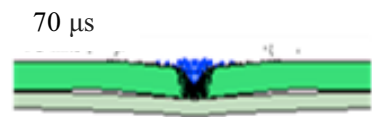

(a)

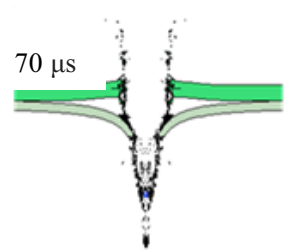

(c)

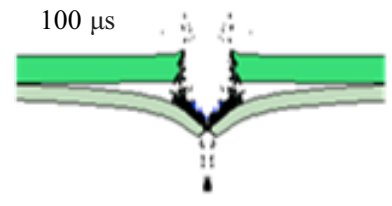

(b)

Fig. 4. Results of calculating the process of interaction of impactors with targets at $\mathrm{T}=-15^{\circ} \mathrm{C}$ with two-layered targets from AcT and AMG-6; $a-V_{o}=500 \mathrm{~m} / \mathrm{s}, \mathrm{b}-\mathrm{V}_{\mathrm{o}}=1000 \mathrm{~m} / \mathrm{s}, \mathrm{c}-\mathrm{V}_{\mathrm{o}}=1500 \mathrm{~m} / \mathrm{s}$. 
At an initial velocity of $1000 \mathrm{~m} / \mathrm{s}$, there is a hole in the asbotextolite layer, and in the layer of AMG-6 - the destruction along the center along the entire thickness. At a coupling velocity of $1500 \mathrm{~m} / \mathrm{s}$ (figure $4 \mathrm{c}$ ), a two-layer obstruction at a time of $70 \mu \mathrm{s}$ has a through hole, and the leading fragments move at a constant velocity equal to $585 \mathrm{~m} / \mathrm{s}$. At the impactor temperature $\mathrm{T}=-50^{\circ} \mathrm{C}$, the leading fragments move at a velocity equal to 635 $\mathrm{m} / \mathrm{s}$.

\section{Conclusions}

Thus, with the use of the developed software complex, numerical investigation of the penetration of targets by ice impactors at various initial temperatures have been carried out. It is established that with the growth of the negative temperature of the shock workers from 15 to 50 degrees, the velocity of the burglary fragments increases from $9.5 \%$ to $11.5 \%$. The results of the calculations can be useful in constructing approximating formulas that connect the inhibitory velocity of the leading fragment of the target with the initial velocity of the damaging element for carrying out an express analysis of the corresponding process of impact interaction of solids.

The work was carried out with the financial support of the Ministry of Education and Science of the Russian Federation.

\section{References}

1. V.P. Glazyrin, Ju.N. Orlov, Ju.N. Orlova, Izvestija vuzov. Fizika, 52, (7) 2 (2009)

2. A. Combescure, Y. Cyuzel-Marmot, J. Fabis, Int. J. Solids Structures, 48, 2779 (2011)

3. V.V. Bogorodskij, V.P. Gavrilo, Led. Fizicheskie svojstva. Sovremennye metody gljaciologii (Gidrometeoizdat, Leningrad, 1980) [In Russian]

4. K.S. Carney, Int. J. Solids Structures, 43, 7820-7839 (2006)

5. A.V. Gerasimov, V.P. Glazyrin Vysokoskorostnoj udar. Modelirovanie i ehksperiment (Izdatel'stvo NTL, Tomsk, 2016) [In Russian]

6. V.P. Glazyrin, M.Ju. Orlov, Ju.N. Orlov, Izvestija vuzov. Fizika, 50, (9) 2 (2007) [In Russian]

7. R.G. Kraus, Earth. Planet. Sci. Lett. 289, 162 (2010)

8. G.N. Bogomolov, V.P. Glazyrin, M.Yu. Orlov, MATEC Web Conf. 72 (2016)

9. V.P. Glazyrin, Ju.N.Orlov, Research J. International Studies, 11(42), 3 (2015) 